\title{
Phonons in a nanoparticle mechanically coupled to a substrate
}

\author{
Kelly R. Patton and Michael R. Geller \\ Department of Physics and Astronomy, University of Georgia, Athens, Georgia 30602-2451
}

(Dated: October 28, 2018)

\begin{abstract}
The discrete nature of the vibrational modes of an isolated nanometer-scale solid dramatically modifies its low-energy electron and phonon dynamics from that of a bulk crystal. However, nanocrystals are usually coupled - even if only weakly - to an environment consisting of other nanocrystals, a support matrix, or a solid substrate, and this environmental interaction will modify the vibrational properties at low frequencies. In this paper we investigate the modification of the vibrational modes of an insulating spherical nanoparticle caused by a weak mechanical coupling to a semi-infinite substrate. The phonons of the bulk substrate act as a bath of harmonic oscillators, and the coupling to this reservoir shifts and broadens the nanoparticle's modes. The vibrational density of states in the nanoparticle is obtained by solving the Dyson equation for the phonon propagator, and we show that environmental interaction is especially important at low frequencies. As a probe of the modified phonon spectrum, we consider nonradiative energy relaxation of a localized electronic impurity state in the nanoparticle, for which good agreement with experiment is found.
\end{abstract}

PACS numbers: $63.22 .+\mathrm{m}, 78.67 . \mathrm{Bf}$

\section{INTRODUCTION}

There is currently great interest in properties of nanometer-scale mechanical systems, such as cantilevers, nanoparticles, and resonators.t Because of the extremely small size and volume-to-surface ratio of these systems, interactions with their surroundings can dramatically alter their properties. In particular, it is well known that the vibrational spectrum of an isolated nanometer-scale crystal, being discrete, is qualitatively different than that of the same bulk material, leading to important changes in any property dependent on the phonon density of states (DOS). The differences between the vibrational DOS in a nanoparticle and a bulk solid are most evident at low frequencies: A spherical nanoparticle with diameter $d$ and characteristic bulk sound velocity $v$ can not support a mode with frequency less than about $2 \pi v / d$. Thus, an acoustic "gap" in the low-energy phonon spectrum is present in contrast with that of the bulk, which has a continuous spectrum down to zero energy. However, mechanical interaction with the environment will modify the discrete nature of the modes.

In an interesting experiment by Yang et al., the phonon DOS deep inside this gap was measured in insulating $\mathrm{Y}_{2} \mathrm{O}_{3}$ nanoparticles. The experiment consisted of nanoparticles whose sizes ranged from 7 to $23 \mathrm{~nm}$ in diameter and was performed by measuring the nonradiative lifetimes of an excited electronic state of a $\mathrm{Eu}^{3+}$ dopant. The lowest supported mode or Lamb mode for nanoparticles of these sizes is about $10 \mathrm{~cm}^{-1}$. At $3 \mathrm{~cm}^{-1}$ the DOS measured was more than 100 times smaller than that of bulk $\mathrm{Y}_{2} \mathrm{O}_{3}\left(\right.$ at $\left.3 \mathrm{~cm}^{-1}\right)$.

In this paper we propose and investigate a mechanism that could be responsible for the observed broadening of the nanoparticles' phonon modes. Several possible broadening mechanisms could be responsible for the observed effect. For example, anharmonicity leads to broadening and, therefore, to a low-energy DOS, but anhar- monicity is ineffective at low energy and was found to be too small to account for the experiment.5 Another possibility could be adsorbed "dirt" on the outside of the nanoparticle. This might lower the $\mathrm{Q}$ factor of the nanoparticle, regarding it as a resonator, reflecting a broadening of the vibrational modes. A third, and in our opinion more likely mechanism, follows from the realization that these nanoparticles are not isolated, but instead were prepared in a powered form. Thus, each nanoparticle is in weak contact with a cluster of other nanoparticles. Because the cluster is relatively large, including several hundred nanoparticles, each nanoparticle is mechanically coupled to a reservoir that has a continuous vibrational DOS at low energy. This interaction broadens the modes and allows phonons in the nanoparticle to escape and be absorbed into the cluster. We investigate the affect this mechanical environmental interaction has on the nanoparticle's phonon DOS.

Because we are only interested in determining the correct origin of broadening, and do not hope to be able to exactly reproduce the experimental results of Ref. 22, we propose the following simplified model: The cluster of nanoparticles is replaced by a semi-infinite elastic substrate, and one nanoparticle is placed in weak mechanical contact with it. The weak contact is imagined to be a few atomic bonds or small neck of material, which we model by a harmonic spring. For simplicity, we take the substrate and the nanoparticle to be made out of the same isotropic elastic material. Because we are interested in the low energy regime, continuum elasticity theory will be used to describe the nanoparticle and the substrate. After defining and analyzing our simplified model, in Sections III through V, we explain in Section VI how the model can be adapted to address the experiment of Ref. 2, and good agreement is obtained.

The simple model we study is related to, but different than, models used to study energy relaxation by molecules adsorbed on surfaces.3 However, in surface 
science the interest is usually in the relaxation of rigid translational motion, rotational motion, or simple internal vibrations of adsorbates. In contrast, we investigate the broadening of complex internal vibrational modes of much larger objects (which are crystalline). Also, our work has much in common with that of Gurevich and Schober 1 where many of the same considerations and modeling were used to study the Lamb-mode decay rate, of nanoparticles caused by both anharmonicity and coupling to an enviroment of other nanoparticles.

\section{NANOPARTICLE AND SUBSTRATE MODEL}

As mentioned in the introduction, the model we study is that of a single nanoparticle in weak mechanical contact with a semi-infinite substrate. Linear elasticity theory will be used to describe the phonons of this system. We assume the nanoparticle and substrate to be made of an isotropic non-polar material. Because we take the nanoparticle and substrate to be made of the same material, we will use the same density $\rho$ and Lamé coefficients $\lambda$ and $\mu$ for both. The Lagrangian for the entire system is given by

$$
L=\int_{\mathbb{V}} d^{3} r\left[\frac{1}{2} \rho\left(\partial_{t} \mathbf{u}\right)^{2}-\frac{1}{2} \lambda u_{i i}^{2}-\mu u_{i j}^{2}\right],
$$

where $\mathbf{u}(\mathbf{r}, t)$ is the displacement field, and

$$
u_{i j} \equiv\left(\partial_{i} u_{j}+\partial_{j} u_{i}\right) / 2
$$

is the strain tensor. $\mathrm{V}$ is the volume of the nanoparticle, substrate, and connecting material, as shown in Fig. 1. Because the Lagrangian density is local, the integration volume in (11) can be split into three independent parts: the nanoparticle, the substrate, and the connecting region. In the limit of weak coupling (diameter $d_{\mathrm{c}}$ of connecting region much smaller than $d$ ), the surface area on the nanoparticle and substrate over which the actual boundary conditions differs from stress-free conditions are negligible, and the Hamiltonian can be written as

$$
H=H_{\text {nano }}+H_{\text {sub }}+\delta H,
$$

where $H_{\text {nano }}$ is the Hamiltonian for an isolated nanoparticle (with stress-free boundaries), $H_{\text {sub }}$ is that for an isolated substrate, and $\delta H$ is the interaction between the two. The connecting region is taken to be a few atomic bonds or small neck of material, as shown in Fig. 1. We further approximate this mechanical coupling to be a harmonic spring potential

$$
\delta H=\frac{1}{2} K:\left[u_{\text {nano }}^{z}\left(\mathbf{r}_{0}\right)-u_{\text {sub }}^{z}\left(\mathbf{r}_{0}\right)\right]^{2}:,
$$

where $K$ is an effective spring constant, and $u_{\text {nano }}^{z}$ and $u_{\mathrm{sub}}^{z}$ are the $z$ components of the displacement field of
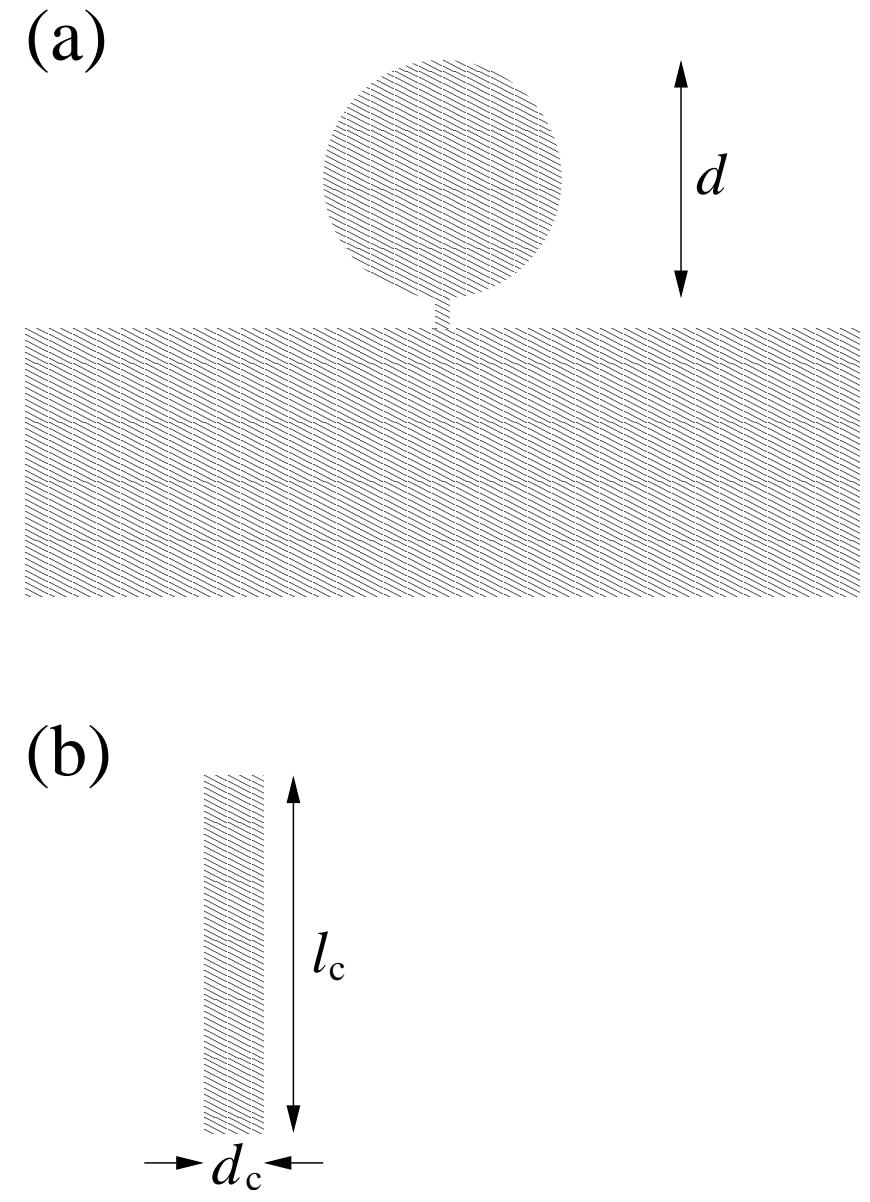

FIG. 1: (a) Model of nanoparticle, substrate, and connecting region. (b) Expanded view of connecting region with dimensions $d_{\mathrm{c}}$ and $l_{\mathrm{c}}$

the nanoparticle and substrate at the point of contact, $\mathbf{r}_{0}$. We take the $z$ direction to be along the upward pointing normal to the substrate surface. The Hamiltonians we will introduce below for $H_{\text {nano }}$ and $H_{\text {sub }}$ are normalordered; therefore, it is necessary to normal order $\delta H$ as well. This operation is denoted by the colons in Eq. (4).

Our analysis will require the vibrational normal modes and spectra of the isolated nanoparticle and semi-infinite substrate, calculated with stress-free boundary conditions. The long-wavelength modes of interest here may be obtained from elasticity theory, to which we now turn.

\section{A. Isolated Nanoparticle}

Here we derive the normal modes of an isolated elastic sphere. The method we shall use is different than (but equivalent to) that used in the classic paper by Lamb, but is better suited for our purposes. The equation of motion of (11) is

$$
\partial_{t}^{2} \mathbf{u}-v_{\mathrm{l}}^{2} \boldsymbol{\nabla}(\boldsymbol{\nabla} \cdot \mathbf{u})+v_{\mathrm{t}}^{2} \boldsymbol{\nabla} \times \boldsymbol{\nabla} \times \mathbf{u}=0,
$$


where $v_{1} \equiv \sqrt{(\lambda+2 \mu) / \rho}$ is the bulk longitudinal sound velocity and $v_{\mathrm{t}} \equiv \sqrt{\mu / \rho}$ is the transverse velocity. To solve Eq. (5) the displacement field can be decomposed into longitudinal and transverse parts,

$$
\mathbf{u}=\mathbf{u}_{1}+\mathbf{u}_{\mathrm{t}}
$$

where

$$
\nabla \times \mathbf{u}_{1}=0
$$

and

$$
\nabla \cdot \mathbf{u}_{\mathrm{t}}=0
$$

With harmonic time dependence, the equation of motion Eq. (5) then separates into two vector Helmholtz equations for the longitudinal and transverse parts,

$$
\left(\nabla^{2}+p^{2}\right) \mathbf{u}_{1}=0, \quad p \equiv \omega / v_{1}
$$

and

$$
\left(\nabla^{2}+q^{2}\right) \mathbf{u}_{\mathrm{t}}=0, \quad q \equiv \omega / v_{\mathrm{t}} .
$$

The longitudinal equation (9) can be solved by introducing a scalar potential

$$
\mathbf{u}_{1}=\nabla \phi^{(p)},
$$

where $\phi^{(p)}$ is a solution of the scalar Helmholtz equation $\left(\nabla^{2}+p^{2}\right) \phi^{(p)}=0$. The transverse equation $(10)$ has two linearly independent solutions, $\mathbf{u}_{\mathrm{t}}=\mathbf{M}$ and $\mathbf{N}$, where

$$
\mathbf{M}=\nabla \phi^{(q)} \times \mathbf{r}
$$

and

$$
\mathbf{N}=\frac{1}{q} \nabla \times \mathbf{M}
$$

Here $\phi^{(q)}$ is a solution of $\left(\nabla^{2}+q^{2}\right) \phi^{(q)}=0$. The prefactor $1 / q$ is included for dimensional convenience. The scalar Helmholtz equations are separable in spherical coordinates and the solutions can be written as

$$
\phi_{l m}(\mathbf{r}) \equiv j_{l}(k r) Y_{l m}(\theta, \varphi), \quad k=p, q
$$

where

$$
j_{l}(x) \equiv \sqrt{\frac{\pi}{2 x}} J_{l+\frac{1}{2}}(x)
$$

is a spherical Bessel function of the first kind (regular at origin) and

$$
Y_{l m}(\theta, \varphi) \equiv(-1)^{m} \sqrt{\frac{2 l+1}{4 \pi} \frac{(l-m) !}{(l+m) !}} P_{l m}(\cos \theta) e^{i m \varphi} .
$$

Here

$$
P_{l m}(x) \equiv\left(1-x^{2}\right)^{\frac{m}{2}} \frac{\partial^{m}}{\partial x^{m}} P_{l}(x)
$$

where $P_{l}(x)$ are Legendre polynomials.

Now we use the $\phi_{l m}$ to construct three linearly independent solutions of (5),

$$
\begin{aligned}
\mathbf{L}_{l m} & \equiv \frac{1}{p} \nabla \phi_{l m}(p r), \\
\mathbf{M}_{l m} & \equiv \nabla \phi_{l m}(q r) \times \mathbf{r} \\
\mathbf{N}_{l m} & \equiv \frac{1}{q} \boldsymbol{\nabla} \times \mathbf{M}_{l m} .
\end{aligned}
$$

The general solution is a linear combination of $\mathbf{L}_{l m}, \mathbf{M}_{l m}$, and $\mathbf{N}_{l m}$

$$
\mathbf{u}(\mathbf{r})=\sum_{l m}\left[a_{l m} \mathbf{L}_{l m}+b_{l m} \mathbf{M}_{l m}+c_{l m} \mathbf{N}_{l m}\right]
$$

Although they are linearly independent, the vector fields $\mathbf{L}_{l m}, \mathbf{M}_{l m}$, and $\mathbf{N}_{l m}$ are not orthogonal in space. However, they can be written in terms of orthogonal vector spherical harmonics $\mathbf{P}_{l m}, \mathbf{B}_{l m}$, and $\mathbf{C}_{l m}$, defined as

$$
\mathbf{P}_{l m}(\Omega) \equiv Y_{l m}(\Omega) \mathbf{e}_{r}
$$

$$
\mathbf{B}_{l m}(\Omega) \equiv \frac{1}{\sqrt{l(l+1)}}\left(\partial_{\theta} Y_{l m}(\Omega) \mathbf{e}_{\theta}+\frac{i m Y_{l m}(\Omega)}{\sin \theta} \mathbf{e}_{\varphi}\right)
$$

$$
\mathbf{C}_{l m}(\Omega) \equiv \frac{1}{\sqrt{l(l+1)}}\left(\frac{i m Y_{l m}(\Omega)}{\sin \theta} \mathbf{e}_{\theta}-\partial_{\theta} Y_{l m}(\Omega) \mathbf{e}_{\varphi}\right)
$$

with the following properties

$$
\int d \Omega \mathbf{X}_{l m}^{*} \cdot \mathbf{X}_{l^{\prime} m^{\prime}}=\delta_{l l^{\prime}} \delta_{m m^{\prime}}
$$

for $\mathbf{X} \in \mathbf{B}, \mathbf{C}, \mathbf{P}$ and

$$
\int d \Omega \mathbf{X}_{l m}^{*} \cdot \mathbf{X}_{l^{\prime} m^{\prime}}^{\prime}=0
$$

for $\mathbf{X} \neq \mathbf{X}^{\prime}$. Expressed in terms of vector spherical harmonics, $\mathbf{L}_{l m}, \mathbf{M}_{l m}$ and $\mathbf{N}_{l m}$ are given by

$$
\mathbf{L}_{l m}=j_{l}^{\prime}(p r) \mathbf{P}_{l m}(\Omega)+\frac{\sqrt{l(l+1)}}{p r} j_{l}(p r) \mathbf{B}_{l m}(\Omega),
$$

$$
\mathbf{M}_{l m}=\sqrt{l(l+1)} j_{l}(q r) \mathbf{C}_{l m}(\Omega)
$$

and

$$
\begin{aligned}
\mathbf{N}_{l m} & =\frac{l(l+1)}{q r} j_{l}(q r) \mathbf{P}_{l m}(\Omega)+\frac{\sqrt{l(l+1)}}{q r} \\
& \times\left[j_{l}(q r)+q r j_{l}^{\prime}(q r)\right] \mathbf{B}_{l m}(\Omega),
\end{aligned}
$$

where prime denotes differentiation with respect to the argument.

Next we impose stress-free boundary conditions

$$
\sigma_{i j} n_{j}=0
$$


at the surface $r=R$ of the nanoparticle. Here $\mathbf{n}$ is an outward pointing normal vector and $\sigma_{i j}$ is the strain tensor. In an isotropic elastic continuum,

$$
\sigma_{i j}=\lambda(\boldsymbol{\nabla} \cdot \mathbf{u}) \delta_{i j}+2 \mu u_{i j}
$$

In spherical coordinates (30) implies

$$
\sigma_{r r}=\sigma_{\theta r}=\sigma_{\varphi r}=0
$$

The three conditions 32 require that

$$
\begin{gathered}
\lambda(\boldsymbol{\nabla} \cdot \mathbf{u})+2 \mu u_{r r}=0, \\
u_{\theta r}=0,
\end{gathered}
$$

and

$$
u_{\varphi r}=0
$$

In terms of the displacement field,

$$
\begin{aligned}
u_{r r} & =\partial_{r} u_{r} \\
u_{\theta r} & =\frac{1}{2}\left(\partial_{r} u_{\theta}-\frac{1}{r} u_{\theta}+\frac{1}{r} \partial_{\theta} u_{r}\right), \\
u_{\varphi r} & =\frac{1}{2}\left(\frac{1}{r \sin \theta} \partial_{\varphi} u_{r}+\partial_{r} u_{\varphi}-\frac{1}{r} u_{\varphi}\right) .
\end{aligned}
$$

The boundary condition equations 32 then become

$$
\begin{aligned}
& a_{l m}\left[-\lambda p j_{l}(p R) Y_{l m}+2 \mu p j_{l}^{\prime \prime}(p R) Y_{l m}\right] \\
& +c_{l m} 2 \mu l(l+1) \mathrm{E} Y_{l m}=0
\end{aligned}
$$

$$
\begin{aligned}
& a_{l m} 2 \mathrm{D} \partial_{\theta} Y_{l m}+b_{l m} i m \mathrm{E} Y_{l m} \csc \theta \\
& +c_{l m} \mathrm{~F} \partial_{\theta} Y_{l m}=0
\end{aligned}
$$

and

$$
\begin{aligned}
& a_{l m} 2 i m \mathrm{D} Y_{l m} \csc \theta+b_{l m} \mathrm{E} \partial_{\theta} Y_{l m} \\
& +c_{l m} i m \mathrm{~F} Y_{l m} \csc \theta=0,
\end{aligned}
$$

where

$$
\begin{aligned}
\mathrm{D} & \equiv \frac{j_{l}^{\prime}(p R)}{R}-\frac{j_{l}(p R)}{p R^{2}}, \\
\mathrm{E} & \equiv \frac{j_{l}^{\prime}(q R)}{R}-\frac{j_{l}(q R)}{q R^{2}}, \\
\mathrm{~F} & \equiv q j_{l}^{\prime \prime}(q R)+\frac{l(l+1)}{q R^{2}} j_{l}(q R)-\frac{2 j_{l}(q R)}{q R^{2}} .
\end{aligned}
$$

Finally, we rewrite (39) though (41) in matrix form as

$$
\left(\begin{array}{ccc}
-\lambda p j_{l}(p R) Y_{l m}+2 \mu p j_{l}^{\prime \prime}(p R) Y_{l m} & 0 & 2 \mu l(l+1) \mathrm{E} Y_{l m} \\
2 \mathrm{D} \partial_{\theta} Y_{l m} & i m \mathrm{E} Y_{l m} \csc \theta & \mathrm{F} \partial_{\theta} Y_{l m} \\
2 i m \mathrm{D} Y_{l m} \csc \theta & \mathrm{E} \partial_{\theta} Y_{l m} & i m \mathrm{~F} Y_{l m} \csc \theta
\end{array}\right)\left(\begin{array}{l}
a_{l m} \\
b_{l m} \\
c_{l m}
\end{array}\right)=0
$$

For a nontrivial solution of (39) through (41) to exist, the determinant of the above matrix must vanish. Taking the determinant and simplifying we find

$$
\left[-\lambda p j_{l}(p R)+2 \mu p j_{l}^{\prime \prime}(p R)\right] \mathrm{E} \mathrm{F}-4 \mu l(l+1) \mathrm{E}^{2} \mathrm{D}=0 .
$$

This implies that either

$$
\mathrm{E}=\frac{j_{l}^{\prime}(q R)}{R}-\frac{j_{l}(q R)}{q R^{2}}=0
$$

or

$$
\left[-\lambda p j_{l}(p R)+2 \mu p j_{l}^{\prime \prime}(p R)\right] \mathrm{F}-4 \mu l(l+1) \mathrm{E} \mathrm{D}=0 .
$$

If (46) is met, then this imposes certain constraints on $a_{l m}, b_{l m}$ and $c_{l m}$, which require $a_{l m}=c_{l m}=0$. This can easily be seen in the above matrix by setting $E=0$. If (47) is met, $b_{l m}$ has to be zero. In conclusion, we have two branches of vibrational modes. The branch in which (46) is satisfied,

$$
\mathbf{u}(\mathbf{r})=b_{l m n} \mathbf{M}_{l m n}(\mathbf{r})
$$

are referred to as the torsional modes, where $n$ specifies the radial quantum number. The other branch is found when (47) is satisfied,

$$
\mathbf{u}(\mathbf{r})=a_{l m n} \mathbf{L}_{l m n}(\mathbf{r})+c_{l m n} \mathbf{N}_{l m n}(\mathbf{r}),
$$

which are the spheroidal modes.

To quantize the vibrational modes we write the displacement field as

$$
\mathbf{u}_{\mathrm{nano}}(\mathbf{r})=\sum_{J} \sqrt{\frac{\hbar}{2 \rho \omega_{J}}}\left[a_{J} \boldsymbol{\Psi}_{J}(\mathbf{r})+a_{J}^{\dagger} \boldsymbol{\Psi}_{J}^{*}(\mathbf{r})\right],
$$

where

$$
J=[\mathrm{S} \text { or T }, n, l, m]
$$

is a label uniquely specifying a nanoparticle eigenmode. The first entry $\mathrm{S}$ or $\mathrm{T}$ specifies whether the mode is in the spheroidal or torsional branch, respectively. $n$ is the radial quantum number and $l$ and $m$ are the usual angular momentum quantum numbers. $a$ and $a^{\dagger}$ are phonon annihilation and creation operators which satisfy the Bose commutation relation

$$
\left[a_{J}, a_{J^{\prime}}^{\dagger}\right]=\delta_{J J^{\prime}}
$$


The $\boldsymbol{\Psi}_{J}$ are vibrational eigenvectors normalized such that

$$
\int_{V}\left|\Psi_{J}(\mathbf{r})\right|^{2} d^{3} r=1
$$

where $V$ is the volume of the nanoparticle. Assuming (without proof) that the modes $\boldsymbol{\Psi}_{J}$ form a complete set,

$$
\sum_{J} \Psi_{J}^{i *}(\mathbf{r}) \Psi_{J}^{j}\left(\mathbf{r}^{\prime}\right)=\delta^{i j} \delta\left(\mathbf{r}-\mathbf{r}^{\prime}\right)
$$

it can easily be shown that $\mathbf{u}$ satisfies the correct equaltime canonical commutation relation with $\boldsymbol{\pi} \equiv \rho \partial_{t} \mathbf{u}$, namely

$$
\left[u^{i}(\mathbf{r}), \pi^{j}\left(\mathbf{r}^{\prime}\right)\right]=i \hbar \delta^{i j} \delta\left(\mathbf{r}-\mathbf{r}^{\prime}\right)
$$

\section{B. Isolated Substrate}

The vibrational modes for a semi-infinite isotropic elastic substrate, with a free surface at the $x y$ plane and extending to infinity in the neorative $z$ direction, were quantized previously by Ezawa: ${ }^{3}$ therefore, the details will be left out here. The displacement field can be written as

$$
\mathbf{u}_{\mathrm{sub}}(\mathbf{r})=\sum_{I} \sqrt{\frac{\hbar}{2 \rho \omega_{I}}}\left[b_{I} \mathbf{f}_{I}(\mathbf{r})+b_{I}^{\dagger} \mathbf{f}_{I}^{*}(\mathbf{r})\right]
$$

where $b$ and $b^{\dagger}$ are the annihilation and creation operators for the substrate phonons. The index $I$, like the index $J$ for the nanoparticle, uniquely specifies a phonon mode for the substrate. $\mathbf{f}_{I}$ are eigenfunctions of (5) subject to stress-free boundary conditions at the $z=0$ plane.

In what follows we will need the spectral density of the isolated substrate which is defined as

$$
N_{\text {sub }}(\mathbf{r}, \omega) \equiv-\frac{1}{\pi} \operatorname{Im} D_{\text {sub }}^{z z}(\mathbf{r}, \mathbf{r}, \omega),
$$

where $D_{\text {sub }}^{i j}\left(\mathbf{r}, \mathbf{r}^{\prime}, \omega\right)$ is the Fourier transform of the retarded phonon Green's function

$$
D_{\mathrm{sub}}^{i j}\left(\mathbf{r}, \mathbf{r}^{\prime}, t\right) \equiv-i \theta(t)\left\langle\left[u_{\mathrm{sub}}^{i}(\mathbf{r}, t), u_{\mathrm{sub}}^{j}\left(\mathbf{r}^{\prime}, 0\right)\right]\right\rangle
$$

of the substrate. The spectral density at the free surface of silicon, regarding it as an isotropic elastic continuum, was calculated in Appendix B of Ref. 9. There we obtained

$$
N_{\mathrm{sub}}(\omega)=C_{\mathrm{Si}} \omega, \quad C_{\mathrm{Si}} \approx 1.4 \times 10^{-46} \mathrm{~cm}^{2} \mathrm{~s}^{2} .
$$

\section{GOLDEN-RULE LIFETIMES}

The relaxation rate or inverse lifetime of the perturbed eigenmodes of the nanoparticle can be calculated using Fermi's golden rule (setting $\hbar=1$ ),

$$
\tau_{J}^{-1}=2 \pi \sum_{\mathrm{f}}|\langle\mathrm{f}|\delta H| \mathrm{i}\rangle|^{2} \delta\left(\omega_{\mathrm{i}}-\omega_{\mathrm{f}}\right),
$$

where the initial and final states are

$$
|\mathrm{i}\rangle=a_{J}^{\dagger}|0\rangle \quad \text { and } \quad|\mathrm{f}\rangle=b_{I}^{\dagger}|0\rangle \text {. }
$$

Using Eqs. (4), (50), and (56) leads to

$$
\tau_{J}^{-1}=\frac{\pi K^{2}}{2 \rho^{2}} \frac{\left|\Psi_{J}^{z}\left(\mathbf{r}_{0}\right)\right|^{2}}{\omega_{J}} \sum_{I} \frac{\left|f_{I}^{z}\left(\mathbf{r}_{0}\right)\right|^{2}}{\omega_{J}} \delta\left(\omega_{J}-\omega_{I}\right) .
$$

Noting that

$$
\sum_{I}\left|f_{I}^{z}\left(\mathbf{r}_{0}\right)\right|^{2} \delta\left(\omega_{J}-\omega_{I}\right)=2 \rho \omega N_{\mathrm{sub}}(\omega),
$$

we obtain (reinstating factors of $\hbar$ )

$$
\tau_{J}^{-1}=\frac{\pi K^{2}}{\hbar \rho} \frac{N_{\mathrm{sub}}\left(\omega_{J}\right)}{\omega_{J}}\left|\Psi_{J}^{z}\left(\mathbf{r}_{0}\right)\right|^{2} .
$$

Using $K=1.0 \times 10^{4} \mathrm{erg} \mathrm{cm}^{-2}$ and $\rho=2.3 \mathrm{~g} \mathrm{~cm}^{-3}$, which are appropriate (see Ref. 9) for a weak link in Si, relaxation rates and $\mathrm{Q}$ factors are given in Table for some low lying modes. The $\mathrm{Q}$ factor is defined here as $\tau$ divided by the period $T$,

$$
\mathrm{Q} \equiv \frac{\tau}{T}=\frac{\hbar \omega_{J}}{2 \pi \gamma}
$$

where $\gamma \equiv \hbar \tau^{-1}$ is an energy width.

The values of the $\mathrm{Q}$ factors we obtain for the low lying modes are incredibly large, reflecting the fact that the reservoir (substrate) is extremely ineffective at absorbing energy at these low frequencies. As we will discuss below in Section VI the lifetimes (and Q factors) for the model considered here cannot directly be compared to the experiment of Ref. 2 without accounting for the difference in sound speeds between a solid Si substrate and a weakly bound nanoparticle cluster, as well as some other less important modifications. There we shall show that the coefficient $C$ in Eq. (59) should be enhanced by about 1000 before making such a comparison, which decreases the $\mathrm{Q}$ factors by this same factor. However, the $\mathrm{Q}$ factors corrected in this way are still huge, and the good agreement with the observed low-frequency DOS (see below) suggests that the $\mathrm{Q}$ factors of the nanoparticles studied experimentally in Ref. 2 are also very large.

In Ref. 10 we used the golden rule result (64) to estimate the phonon DOS at low energies. This is achieved by replacing, in accordance with Fermi's golden rule, each discrete mode in the isolated nanoparticle by a Lorentzian with a width given by (64). (More precisely, this amounts to approximating the energy-dependent phonon self-energy for each mode $J$ with its value at $\omega=\omega_{J}$, a procedure often called the quasiparticle-pole approximation.) However, this procedure is unreliable at low energies because the actual line-shapes of the broadened modes are non-Lorentzian in the tails. Nevertheless, we obtained a DOS at $3 \mathrm{~cm}^{-1}$ that was only 20 times smaller than that observed.11 
TABLE I: A few representative relaxation rates and Q factors. Note that only the $m=0$ spheroidal modes are broadened by the interaction.

\begin{tabular}{lccc}
\hline \hline$(\mathrm{S}, l, m, n)$ & $\omega\left(\mathrm{rad} \mathrm{s}^{-1}\right)$ & $\tau^{-1}\left(\mathrm{~s}^{-1}\right)$ & $\mathrm{Q}$ factor \\
\hline$(\mathrm{S}, 2,0,1)$ & $3.5 \times 10^{12}$ & $2.5 \times 10^{-4}$ & $2.2 \times 10^{15}$ \\
$(\mathrm{~S}, 1,0,1)$ & $4.9 \times 10^{12}$ & $1.0 \times 10^{-8}$ & $7.8 \times 10^{19}$ \\
$(\mathrm{~S}, 0,0,1)$ & $1.1 \times 10^{13}$ & $3.6 \times 10^{-4}$ & $4.9 \times 10^{15}$ \\
$(\mathrm{~S}, 0,0,2)$ & $1.7 \times 10^{13}$ & $1.1 \times 10^{-3}$ & $2.5 \times 10^{15}$ \\
\hline \hline
\end{tabular}

\section{MANY-BODY THEORY OF THE DOS}

\section{A. Local DOS}

To leading order in the electron-phonon interaction strength, the electronic population relaxation rate due to phonon emission (for example, as measured in Ref. 2) is given by Fermi's golden rule, which states the rate (for a deformation potential electron-phonon interaction) is proportional to the square of the electron-phonon coupling strength times the phonon DOS. In a translationally invariant system the DOS does not have any position dependence, but in a nanoparticle one must distinguish between the "global" DOS (the DOS relevant for thermodynamics) and the local eigenfunction-weighted DOS, which is the one that determines phonon emission rate. We will call this position-dependent DOS the local DOS, and denote it by $g(\mathbf{r}, \omega)$. The precise definition of $g(\mathbf{r}, \omega)$ will be given below.

From a theoretical point-of-view, the quantity describing the local vibrational dynamics in the nanoparticle is the (retarded) phonon Green's function

$$
D_{\mathrm{R}}^{i j}\left(\mathbf{r}, \mathbf{r}^{\prime}, t\right) \equiv-i \theta(t)\left\langle\left[u^{i}(\mathbf{r}, t), u^{j}\left(\mathbf{r}^{\prime}, 0\right)\right]\right\rangle_{H},
$$

where

$$
\langle\cdot\rangle_{H} \equiv \frac{\operatorname{Tr}\left(e^{-\beta H} \cdot\right)}{\operatorname{Tr} e^{-\beta H}},
$$

and with the Hamiltonian given by

$$
H=H_{0}+\delta H \text {. }
$$

Here $H_{0}$ is the Hamiltonian of the isolated nanoparticle and substrate,

$$
H_{0}=\sum_{J} \omega_{J} a_{J}^{\dagger} a_{J}+\sum_{I} \omega_{I} b_{I}^{\dagger} b_{I},
$$

and, as mentioned in Section II, $\delta H$ is a harmonic spring potential given in Eq. (4).

In this section the phonon Green's function $D$ always refers to the nanoparticle, and the label "nano" will be suppressed. The imaginary part of the Fourier transform of $D_{\mathrm{R}}^{i j}(\mathbf{r}, \mathbf{r}, t)$ defines the nanoparticle's phonon spectral density

$$
N^{i j}(\mathbf{r}, \omega) \equiv-\frac{1}{\pi} \operatorname{Im} D_{\mathrm{R}}^{i j}(\mathbf{r}, \mathbf{r}, \omega)
$$

For an electron system (or any system of particles), the spectral density defined above is precisely the local DOS. However, because the elasticity equation of motion (5) is second order in time, the spectral density and DOS (both local and global) differ by a factor of $2 \rho \omega$. In addition, the vibrational spectral density (70) is a tensor, whereas the phonon emission rate probes some couplingconstant-weighted sum of tensor elements. Because we are ascribing the observed reduction in phonon emission (in going from bulk to nanoparticle) to a reduction in the local DOS, our results are not sensitive to the precise way in which a scalar quantity is constructed from the tensor, as long as the same measure is used in both the nanoparticle and bulk. It will be most convenient to investigate the trace of the local DOS tensor. Therefore, the quantity we calculate in this paper is

$$
g(\mathbf{r}, \omega) \equiv 2 \rho \omega \sum_{i=1}^{3} N^{i i}(\mathbf{r}, \omega),
$$

which we shall refer to as the local DOS. $g(\mathbf{r}, \omega)$ characterizes the number of states per unit energy per unit volume near position $\mathbf{r}$. In a bulk material with Debye spectrum, (71) reduces at low frequency to

$$
g(\mathbf{r}, \omega)=\frac{\omega^{2}}{2 \pi^{2}}\left(\frac{1}{v_{1}^{3}}+\frac{2}{v_{\mathrm{t}}^{3}}\right),
$$

independent of r. Eq. (72) is the well-known Debye formula for the vibrational DOS of a crystal.

The local DOS $g(\mathbf{r}, \omega)$ controls the phonon emission rate for an impurity atom sitting at position $\mathbf{r}$. Although the impurity locations in a real nanoparticle are assumed to be random, dopants near the surface are known to be optically inactive; hence, the experiments (including that of Ref. 2) do not probe the phonon DOS near the nanoparticle surface. Therefore, we introduce a particular volume-averaged DOS

$$
\bar{g}(b, \omega) \equiv \frac{\int_{r \leq b} d^{3} r g(\mathbf{r}, \omega)}{\frac{4}{3} \pi b^{3}},
$$

which characterizes the average $g(\mathbf{r}, \omega)$ within a sphere of radius $b$. In the limit $b \rightarrow R$, in which case the local DOS is averaged over the full nanoparticle volume, we obtain the global (or thermodynamic) DOS, which, for an isolated nanoparticle, is

$$
\bar{g}(R, \omega)=\frac{1}{V} \sum_{J} \delta\left(\omega-\omega_{J}\right) .
$$

Physically, we expect $b$ to be somewhere between $R / 2$ and $R$.

\section{B. Perturbative Calculation of the Local DOS}

The retarded Green's function (66) for the nanoparticle can be obtained by calculating the Euclidean time- 
ordered (or imaginary time) Green's function defined by

$$
D^{i j}\left(\mathbf{r}, \mathbf{r}^{\prime}, \tau\right)=-\left\langle T u^{i}(\mathbf{r}, \tau) u^{j}\left(\mathbf{r}^{\prime}, 0\right)\right\rangle_{H} .
$$

In the interaction representation,

$$
D^{i j}\left(\mathbf{r}, \mathbf{r}^{\prime}, \tau\right)=-\frac{\left\langle T u^{i}(\mathbf{r}, \tau) u^{j}\left(\mathbf{r}^{\prime}, 0\right) e^{-\int_{0}^{\beta} \delta H\left(\tau^{\prime}\right) d \tau^{\prime}}\right\rangle_{H_{0}}}{\left\langle e^{-\int_{0}^{\beta} \delta H\left(\tau^{\prime}\right) d \tau^{\prime}}\right\rangle_{H_{0}}},
$$

where the expectation values are with respect to $H_{0}$. By expanding the exponentials to leading order in the perturbation and Fourier transforming, (76) can be written as

$$
\begin{aligned}
D^{i j}\left(\mathbf{r}, \mathbf{r}^{\prime}, \omega\right) & =D_{0}^{i j}\left(\mathbf{r}, \mathbf{r}^{\prime}, \omega\right)+\sum_{k l} \int D_{0}^{i k}\left(\mathbf{r}, \mathbf{r}^{\prime \prime}, \omega\right) \\
& \times \Pi^{k l}\left(\mathbf{r}^{\prime \prime}, \mathbf{r}^{\prime \prime \prime}, \omega\right) D_{0}^{l j}\left(\mathbf{r}^{\prime \prime \prime}, \mathbf{r}^{\prime}, \omega\right) d^{3} r^{\prime \prime} d^{3} r^{\prime \prime \prime}
\end{aligned}
$$

where

$$
D_{0}^{i j}\left(\mathbf{r}, \mathbf{r}^{\prime}, \omega\right)=\sum_{J} \frac{\Psi_{J}^{i}(\mathbf{r}) \Psi_{J}^{j *}\left(\mathbf{r}^{\prime}\right)}{2 \rho \omega_{J}}\left[\frac{1}{i \omega-\omega_{J}}-\frac{1}{i \omega+\omega_{J}}\right]
$$

is the free propagator and $\Pi^{i j}$ is the leading order selfenergy, given at zero temperature byt

$$
\begin{aligned}
\Pi^{i j}\left(\mathbf{r}, \mathbf{r}^{\prime}, \omega\right) & =\frac{K^{2}}{2 \rho} \sum_{\mathrm{I}} \frac{\left|f_{\mathrm{I}}^{z}\left(\mathbf{r}_{0}\right)\right|^{2}}{\omega_{\mathrm{I}}}\left[\frac{1}{i \omega-\omega_{\mathrm{I}}}-\frac{1}{i \omega+\omega_{\mathrm{I}}}\right] \\
& \times \delta^{i z} \delta^{j z} \delta\left(\mathbf{r}-\mathbf{r}_{0}\right) \delta\left(\mathbf{r}^{\prime}-\mathbf{r}_{0}\right) .
\end{aligned}
$$

The $\mathbf{f}_{\mathrm{I}}(\mathbf{r})$ are the substrate eigenfunctions discussed in Section II, and $\mathbf{r}_{0}$ is the point at which the nanoparticle is connected to the substrate. Retarded quantities are obtained by analytically continuing $i \omega \rightarrow \omega+i 0^{+}$.

To calculate the local DOS (71) we need to solve the Dyson equation for the nanoparticle Green's function, written symbolically as

$$
D=D_{0}+D_{0} \Pi D .
$$

A solution to (80) can be obtained by introducing matrix representations for $D, D_{0}$, and $\Pi$, in which case

$$
D=\left(D_{0}^{-1}-\Pi\right)^{-1} \text {. }
$$

The matrix representation we use is defined by

$$
O\left(J, J^{\prime}, \omega\right) \equiv \sum_{i j} \int_{V} d^{3} r d^{3} r^{\prime} \Psi_{J}^{i *}(\mathbf{r}) \Psi_{J^{\prime}}^{j}\left(\mathbf{r}^{\prime}\right) O^{i j}\left(\mathbf{r}, \mathbf{r}^{\prime}, \omega\right),
$$

where $O=D, D_{0}$, or $\Pi$. In (82) the integration is over the volume $V$ of the nanoparticle, and the $\boldsymbol{\Psi}_{J}(\mathbf{r})$ are the nanoparticle eigenfunctions. The inverse transformation is

$$
O^{i j}\left(\mathbf{r}, \mathbf{r}^{\prime}, \omega\right)=\sum_{J J^{\prime}} \Psi_{J}^{i}(\mathbf{r}) O\left(J, J^{\prime}, \omega\right) \Psi_{J^{\prime}}^{j *}\left(\mathbf{r}^{\prime}\right) .
$$

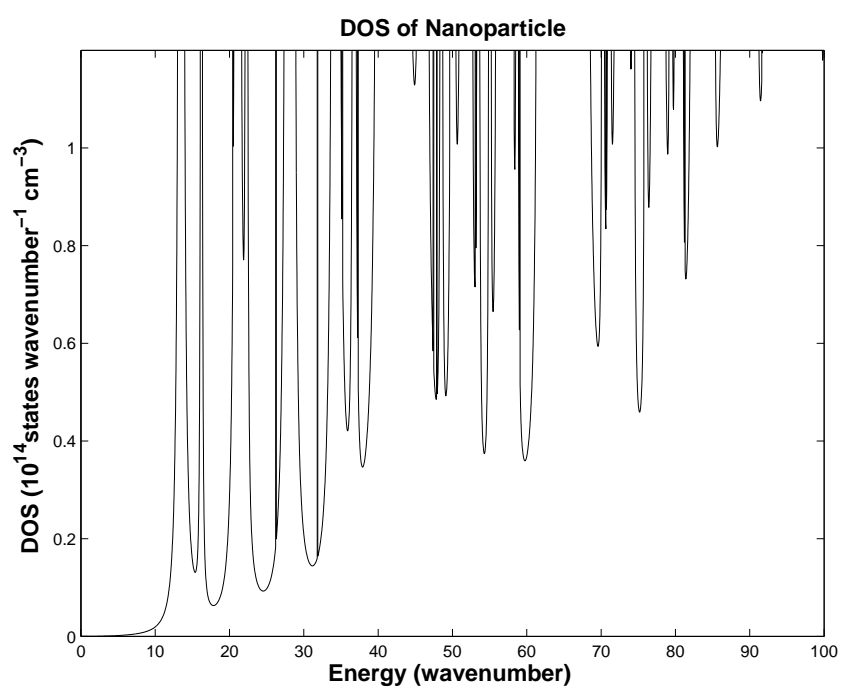

FIG. 2: The phonon DOS, given in states per wavenumber per $\mathrm{cm}^{3}$, of a $10 \mathrm{~nm}$ Si nanoparticle, weakly coupled to a semi-infinite substrate.

A nanoparticle with a diameter of $10 \mathrm{~nm}$ has approximately 8,000 atoms in it, thus, there are roughly 24,000 acoustic vibrational modes. By knowing the number of modes, a Debye energy can be defined: the Debye energy is the energy at which there are 24,000 elasticity-theory modes that lie below in energy. For our nanoparticle, the Debye energy is about $240 \mathrm{~cm}^{-1}$. The Debye energy cutoff truncates the Hilbert space, which leads to finite-size matrices. 2 This enables every mode $J$ of the nanoparticle to be included in the calculation of the Green's function (81).

\section{RESULTS}

In this section we present our results for the phonon DOS in a $10 \mathrm{~nm}$ Si nanoparticle, obtained by solving the Dyson equation (80) for the phonon Green's function, as explained above. As we have discussed, the DOS $g(\mathbf{r}, \omega)$, defined in (71), is a local quantity that varies with position within the nanoparticle, and, as mentioned in the previous section, the quantity we are interested in is $\bar{g}(b, \omega)$, which is $g(\mathbf{r}, \omega)$ averaged over a sphere of radius $b$ centered at the center of the nanoparticle. Because we have found no significant dependence of $\bar{g}(b, \omega)$ on $b$, for the physically relevant values of $b(R / 2<b<R)$, we plot $\bar{g}(R, \omega)$. As stated above in Section IVA, $\bar{g}(R, \omega)$ is the global phonon DOS in the nanoparticle.

For simplicity we assume both the nanoparticle and the substrate to be made of $\mathrm{Si}$; this allows us to use the surface spectral density (59) calculated in Appendix B of Ref. 9, where $\mathrm{Si}$ is treated as an isotropic elastic continuum with longitudinal and transverse sound velocities

$$
\begin{aligned}
& v_{\mathrm{l}}=8.5 \times 10^{5} \mathrm{~cm} \mathrm{~s}^{-1}, \\
& v_{\mathrm{t}}=5.9 \times 10^{5} \mathrm{~cm} \mathrm{~s}^{-1},
\end{aligned}
$$




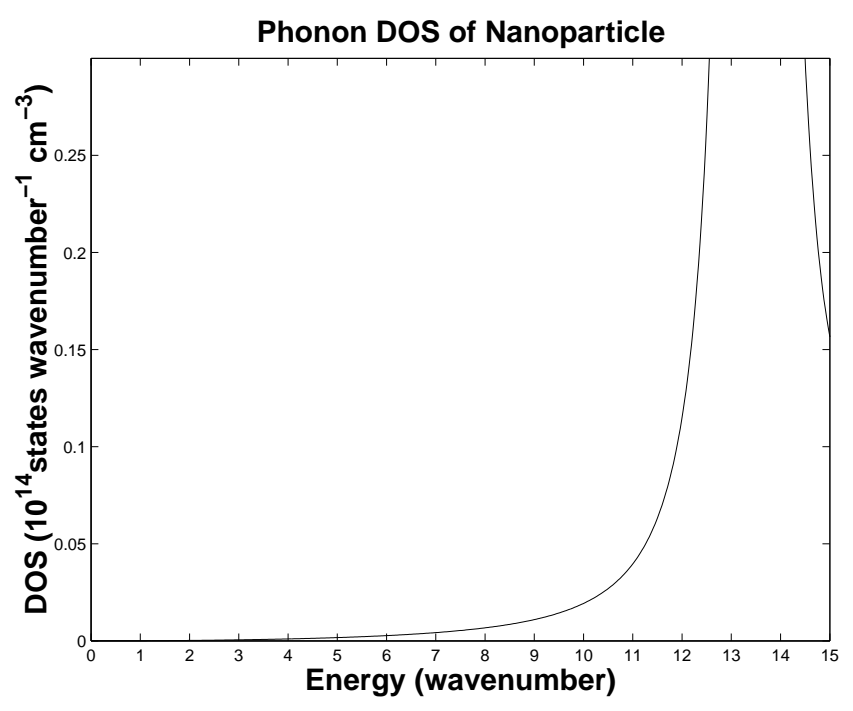

FIG. 3: Vibrational DOS at low energies.

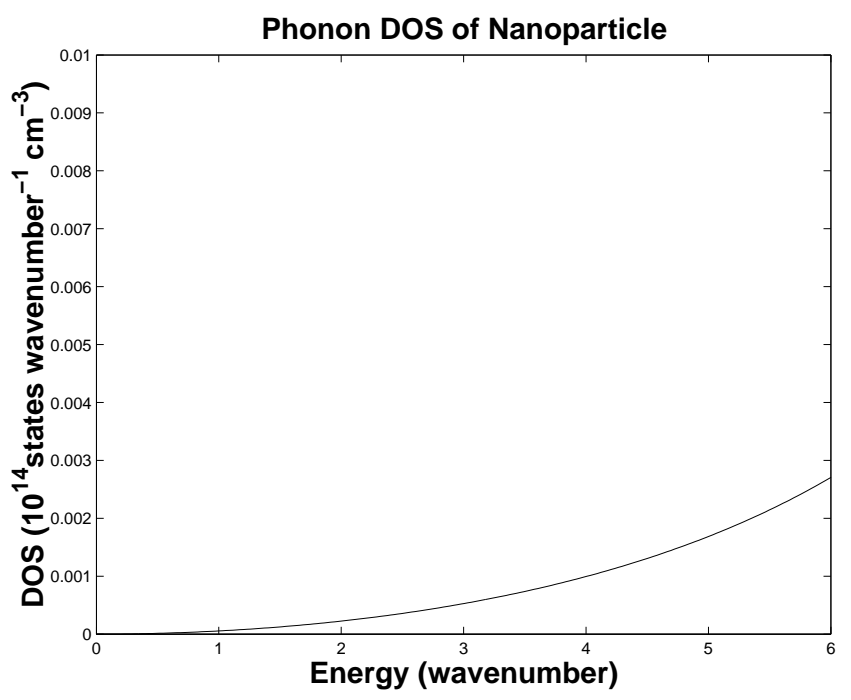

FIG. 4: Expanded view of the low-energy DOS. Note that the DOS vanishes at zero energy, as expected.

and mass density $\rho=2.3 \mathrm{~g} \mathrm{~cm}^{-3}$. In the final section of this paper, where we compare our results to the experiment of Ref. 2, we will introduce an important correction to account for the differences between a solid Si substrate and a nanoparticle cluster.

In Fig. 目 the global DOS $\bar{g}(R, \omega)$ of a $10 \mathrm{~nm}$ diameter nanoparticle is given up to $100 \mathrm{~cm}^{-1}$. The modes above $100 \mathrm{~cm}^{-1}$ were included in the calculation, but the longwavelength approximation of elasticity theory becomes invalid at high energy. Thus, only the lower part of the spectrum is shown. Fig. 3 shows the low-energy phonon DOS up to about $15 \mathrm{~cm}^{-1}$. The large peak on the right side is the well-known Lamb mode. The phonon DOS at 3 $\mathrm{cm}^{-1}$ is approximately $4.9 \times 10^{10}$ states per wavenumber per $\mathrm{cm}^{3}$.

\section{COMPARISON WITH EXPERIMENT}

In this section we compare our results with the experiment of Ref. 2, where the one-phonon emission rate (and, therefore, the phonon DOS at $3 \mathrm{~cm}^{-1}$ ) in a cluster of $\mathrm{Y}_{2} \mathrm{O}_{3}$ nanoparticles was observed to be $8.2 \times 10^{-3}$ times that in bulk $\mathrm{Y}_{2} \mathrm{O}_{3}$. In particular, the excited ${ }^{5} \mathrm{D}_{1}$ (II) state of $\mathrm{Eu}^{3+}$ in the nanoparticles had a phonon-emission lifetime of $27 \mu \mathrm{s}$, compared with a bulk value of $221 \mathrm{~ns}$. In order to make a comparison of our results to that of the experiment, two modifications of our calculation have to be performed.

In our model, the cluster of nanoparticles has been replaced by a solid substrate. However, the spectral density (59) of the substrate, which at long wavelengths, is determined by the sound speeds and mass density of $\mathrm{Si}$, is much smaller than that of the nanoparticle cluster. Treating the long-wavelength modes of the cluster with elasticity theory (or, even simpler, approximating the random cluster by an ordered cubic lattice), shows that the spectral density (59) should be replaced by (the subscript "cl" referring to cluster)

$$
N_{\mathrm{cl}}(\omega)=C_{\mathrm{cl}} \omega
$$

where 13

$$
C_{\mathrm{cl}}=\frac{v_{\mathrm{Si}}^{3} \rho_{\mathrm{Si}}}{v_{\mathrm{cl}}^{3} \rho_{\mathrm{cl}}} C_{\mathrm{Si}}
$$

Here $v_{\mathrm{cl}}$ is a characteristic sound speed in the cluster and $\rho_{\mathrm{cl}}$ is its mass density. The $1 / v_{\mathrm{cl}}^{3}$ dependence in (86) comes from the well-known velocity dependence of the Debye DOS, and the $1 / \rho_{\mathrm{cl}}$ factor comes from the definition of spectral density [see discussion following Eq. (70)]. Approximating the cluster by an ordered cubic array with lattice constant $d$ (the nanoparticle diameter), yields

$$
v_{\mathrm{cl}} \approx \sqrt{\frac{K}{M}} d
$$

and

$$
\rho_{\mathrm{cl}} \approx \frac{\pi}{6} \rho_{\mathrm{Si}},
$$

where $K$ is the effective spring constant connecting the nanoparticles, given after Eq. (64), and

$$
M=\frac{4}{3} \pi \rho_{\mathrm{Si}}\left(\frac{d}{2}\right)^{3}
$$

is the mass of one nanoparticle. Using $d=13 \mathrm{~nm}$ (the mean diameter in Ref. 2), we obtain an enhancement factor

$$
\frac{C_{\mathrm{cl}}}{C_{\mathrm{Si}}}=9.8 \times 10^{2} .
$$

This factor increases the nanoparticle DOS (at frequencies below the Lamb mode) by nearly three orders of magnitude. 
There are several other marginally important corrections, most of which will be ignored, and one that will be included just for completeness: In the model analyzed above, the nanoparticle was connected to its surroundings by only a single contact point, whereas a nanoparticle in a cluster most likely has more than one connection. As the number of contacts increases, this simply scales the DOS (away from the peaks) linearly with the number of contact points. Conservatively, we expect that the multiple contact points present in the real system will increase the decay rate of the nanoparticles' modes, and hence the phonon DOS well below the Lamb mode, by a factor of two.

The following additional corrections have also been considered and were found not to be significant (and are not included in our final results):

(i) The actual experiment of Ref. 2 was done on an ensemble of nanoparticles with mean diameter $13 \mathrm{~nm}$ and standard deviation of $5 \mathrm{~nm}$. To understand the effects of this size distribution, we have calculated the DOS at $3 \mathrm{~cm}^{-1}$ averaged over a Gaussian distribution of diameters centered at $10 \mathrm{~nm}$. Even for very wide distributions (standard deviation up to $8 \mathrm{~nm}$ ), the ensemble averaged DOS $3 \mathrm{~cm}^{-1}$ is increased by no more than a factor of two 14 In addition, the correction for re-centering the size distribution from $10 \mathrm{~nm}$ to the experimentally observed $13 \mathrm{~nm}$ average size, leads to corrections of order unity.

(ii) Our calculations were done for a nanoparticle and substrate made of $\mathrm{Si}$, while the experiment was done on $\mathrm{Y}_{2} \mathrm{O}_{3}$ nanoparticles, which, of course, has different mass density and sound velocities. The differences in sound speeds and mass only shift around the modes of the nanoparticle by a small amount; this has the same effect as a small change in the diameter of the nanoparticles, which we have found to be negligible. As for the substrate (or more precisely the replacement of the substrate with a cubic lattice of nanoparticles), the change in mass density does effect the spectral function (59) by changing the velocity (87) and mass density (88), but this change is only of order unity.

(iii) The nanoparticles of Ref. 2 were immersed in He, either liquid (for $\mathrm{T}<4.21 \mathrm{~K}$ ) or gas $(\mathrm{T}>4.21 \mathrm{~K}$ ). However, the results were found not to change through the liquid-gas transition, presumably because of the large sound-speed mismatch between superfluid $\mathrm{He}$ and $\mathrm{Y}_{2} \mathrm{O}_{3}$. Therefore, we have ignored the presence of $\mathrm{He}$ in our theory.

(iv) The experiment of Ref. 2 2 was done at temperatures between 1.5 and $10 \mathrm{~K}$ (excluding the interval 2.17 to $4.21 \mathrm{~K}$ ), whereas our calculations assume zero temperature. The effect of finite temperature is to stimulate phonon emission into the bath (i.e. substrate or cluster). However, this is not important until the Bose distribution function of the bath at the Lamb mode frequency (approximately $13 \mathrm{~cm}^{-1}$ ) becomes of order unity, which does not occur until the temperature exceeds about 18 $\mathrm{K}$.

Taking into account the first two modifications, and ignoring the rest, we obtain a $3 \mathrm{~cm}^{-1}$ DOS given by

$$
\begin{aligned}
\bar{g}\left(R, 3 \mathrm{~cm}^{-1}\right) & =4.9 \times 10^{10} \frac{\text { states }}{\text { wavenumber } \mathrm{cm}^{3}} \\
& \times 980 \times 2 \\
& =9.6 \times 10^{13} \frac{\text { states }}{\text { wavenumber } \mathrm{cm}^{3}} .
\end{aligned}
$$

The DOS of bulk Si is given by

$$
\sum_{\lambda} \frac{\epsilon^{2}}{2 \pi^{2} \hbar^{3} v_{\lambda}^{3}}=3.9 \times 10^{15} E^{2} \frac{\text { states }}{\text { wavenumber } \mathrm{cm}^{3}}
$$

where $E$ is the energy in wavenumbers. Thus, the theoretical ratio of the DOS of the nanoparticle to that of the bulk material at $3 \mathrm{~cm}^{-1}$ is $2.7 \times 10^{-3}$. As stated above, the experimental ratio of nanoparticle to bulk DOS was found to be approximately $8.2 \times 10^{-3}$. The agreement between our theory and the experiment of Ref. 2 is excellent considering the simplicity and robustness of our model. We conclude that the low-energy phonon DOS observed in Ref. 2 is consistent with our enviromental broadening mechanism.

\section{CONCLUSIONS}

Motivated by an interesting experiment measuring the low-energy phonon DOS in a insulating nanoparticle, we have thoroughly investigated a simplified model of a single nanoparticle weakly coupled to its environment, a semi-infinite substrate. The environmental interactions were found to significantly affect the DOS at energies below the Lamb mode.

Additionally, we have used the results of our model to predict the effect of environmental interaction in a cluster of nanoparticles like that studied in Ref. 2. Although it is necessary to estimate the value of several quantities appearing in the model, we believe that we can do this accurately enough to obtain a final result that is correct at the order-of-magnitude level, with no free parameters. Because our results for the $3 \mathrm{~cm}^{-1}$ DOS is only about three times smaller than that observed in Ref. 2, our broadening mechanism and the resulting phonon spectrum is clearly consistent with that experiment.

\section{ACKNOWLEDGMENTS}

This work was supported by the National Science Foundation under CAREER Grant No. DMR-0093217, and by the Research Corporation. Is is a pleasure to thank Steve Lewis, Vadim Markel, Richard Meltzer, Mark Stockman, and Ho-Soon Yang for useful discussions, and Patrick Sprinkle for help with the numerics. We would especially like to thank Bill Dennis for his help with every aspect of this work. 
1 For introductory accounts see M. Roukes, Sci. Am. Sept. 2001, p.48, and D. Bishop, P. Gammel and C. R. Giles, Phys. Today 54 No. 10 2001, p. 38.

2 H. S. Yang, S. P. Feofilov, D. K. Williams, J.C. Milora, B. M. Tissue, R.S. Meltzer, and W.M. Dennis, Physica B 263, (1999).

3 A. Zangwill, Physics at Surfaces (Cambridge, New York, 1988).

4 V. L. Gurevich and H. R. Schober, Phys. Rev. B 57, 11295 (1998).

5 V.A. Markel and M. R. Geller, J. Phys. Condens. Matter 12, 7569 (2000).

${ }^{6}$ H. Lamb, Proc. Math. Soc. London 13 (1882).

7 Throughout this work we use $I$ to label the vibrational modes of the substrate and $J$ for the nanoparticle.

${ }^{8}$ H. Ezawa, Ann. Phys. 67, 438 (1971).

9 K. R. Patton and M. R. Geller, Phys. Rev. B 64, 155320 (2001).
10 K. R. Patton and M. R. Geller, J. Lumin. 94, 747 (2001).

11 This agreement is fortuitous, however, because we did not account for the modified sound velocity of the bath. After this modification is made, the golden-rule estimate for the low-frequency DOS is too large (by a factor of 50 at 3 $\mathrm{cm}^{-1}$ ), as expected.

12 Each such double-precision complex matrix requires about 9 GB of memory.

13 For Si we use the branch-averaged velocity

$$
v_{\mathrm{Si}}=\left(\frac{1}{3} \sum_{\lambda} \frac{1}{v_{\lambda}^{3}}\right)^{-1 / 3}=6.4 \times 10^{5} \mathrm{~cm} \mathrm{~s}^{-1} .
$$

14 We note, however, that when the Lamb mode frequency becomes resonant with the electronic two-level system, the electronic relaxation rate is not simply related to the phonon DOS. 